\title{
An OAI-based Filtering Service for CITIDEL from NDLTD
}

\author{
Baoping Zhang, Marcos André Gonçalves, and Edward A. Fox \\ Digital Library Research Laboratory, Virginia Tech \\ Blacksburg, VA 24061 USA \\ $\{$ bzhang, mgoncalv, fox\}@vt.edu
}

\begin{abstract}
One goal of the Computing and Information Technology Interactive Digital Educational Library (CITIDEL) is to maximize the number of computing-related resources available to computer science scholars and practitioners through it. In this paper, we describe a set of experiments designed to help this goal by adding to CITIDEL a sub-collection of computing related electronic theses and dissertations (ETDs) automatically extracted from the Networked Digital Library of Theses and Dissertations (NDLTD) OAI Union Catalog. We analyze the metadata quality of the NDLTD OAI Union Catalog and describe three different experiments that combine different sources of evidence to improve the accuracy in filtering out the computing related entries.
\end{abstract}

\section{Introduction}

CITIDEL [1] is part of the Collections Track activities in the National STEM (Science, Technology, Engineering, and Mathematics education) Digital Library (NSDL). In particular, CITIDEL operates and maintains the "computing" content of the digital library that includes information systems, computer science, information science, information technology, software engineering, computer engineering, and other computing-related fields. This work focuses on enhancing the current coverage of the CITIDEL collection by automatically extracting computing-related records from the NDLTD [2-5] collection and adding them into CITIDEL.

\subsection{Background and Motivation}

The Open Archives Initiative [6] Protocol for Metadata Harvesting provides an application-independent interoperability framework based on metadata harvesting. There are two classes of participants in the OAI-PMH framework:

1. Data Providers administer systems that support the OAI-PMH as a means of exposing metadata;

2. Service Providers use metadata harvested via the OAI-PMH as a basis for building value-added services. 
A repository is a network accessible server that can process the six OAI-PMH requests. A repository is managed by a data provider to expose metadata to harvesters. The NDLTD OAI Union Catalog [5] is an example of a service built by harvesting metadata via the Open Archives Initiative [6] Protocol from Open Archives of electronic theses and dissertations worldwide. At the time of the experiments there were 7507 records in the Union Catalog, but we expect that, through partnering with OCLC and because of its support by providing data from WorldCat, there eventually will be millions of records.

In order to achieve the CITIDEL goal of maximum coverage of computing related information, we have designed and conducted a set of experiments that harvest metadata in Dublin Core [7] format from the OAI Union Catalog and then filter out the computing related entries.

One way to filter out computing entries from the whole NDLTD collection would be to look at the contributor fields of each record (in the case of ETDs where this entry corresponds to mentors and/or committee members). For example, if most of the contributors are computer scientists, one could expect that this record is computing related. Another way is to look at the subject fields.

Figure 1 shows the number of entries which have subject field(s) and contributor field(s) for the whole NDLTD collection. Figure 2 shows this for each university (site) in the collection, in a series of groups, organized according to size of collection. Figure 1 shows that only about $65 \%$ of the entries have contributor fields while about $92 \%$ have subject fields. Figure 2 also shows the inconsistency of using contributor and subject fields across universities. For example, all entries from USF have both contributor and subject fields while all entries from MIT are missing both fields. All entries from LSU have subject field but no contributor field. And some but not all entries from WUW have subject fields and some but not all entries from Duisburg have contributor fields. This analysis shows that the filtering task using only contributor is impossible. To address those entries with missing contributor fields, we need to look at other sources of evidence.

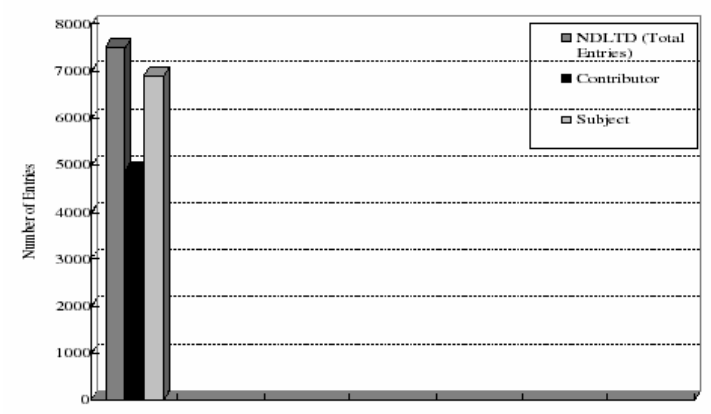

Fig. 1. Entry counts with contributor field(s) and subject fields

Though the subject fields are most useful overall in filtering, their sole use is prevented by a number of factors. The first factor, according to Figure 2, is that many records at several sites are missing subject fields. If we simply filter using subject information, we would exclude those records. The second factor is the inconsistency 


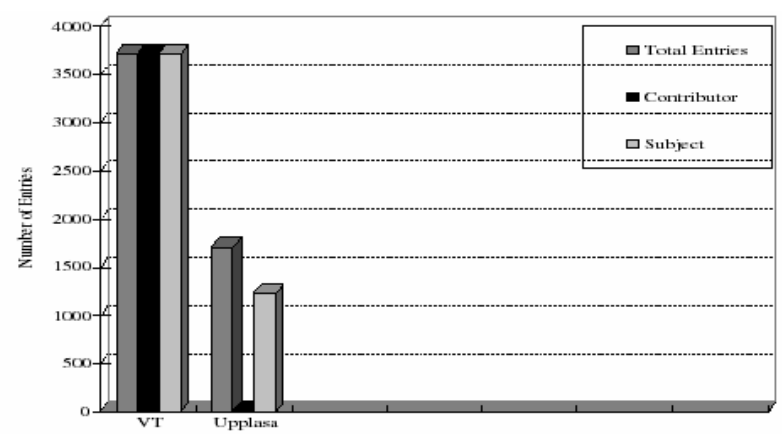

Fig. 2(a). Entry counts with contributor field(s) and subject field(s) for different sites

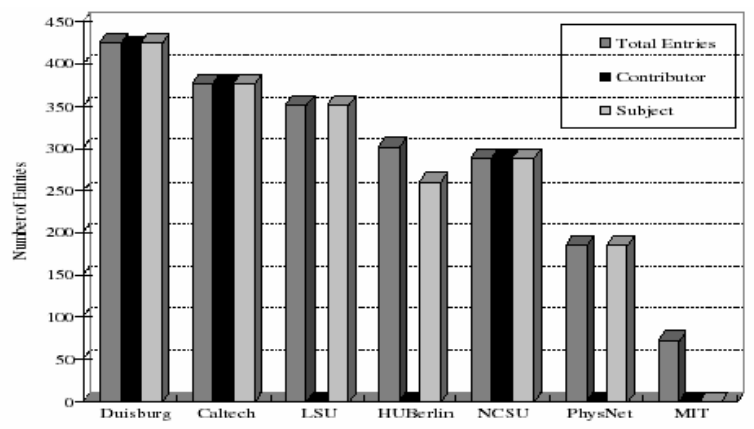

Fig. 2(b). Entry counts with contributor field(s) and subject field(s) for different sites

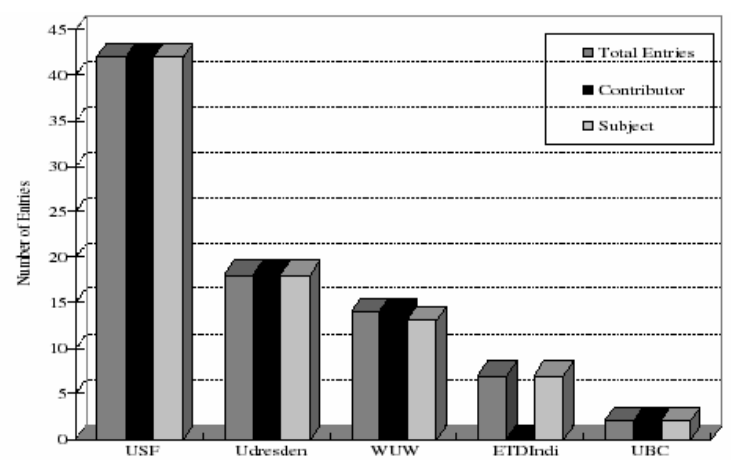

Fig. 2(c). Entry counts with contributor field(s) and subject field(s) for different sites

in use of subjects at several universities as an indicator of topical field. For example, 
at Virginia Tech, subject represents department name, so for a computing related record, the subject would be "computer science". This subject entry clearly indicates the broad topic of the record. But this practice is not universal among universities. For example, in a record from Uppsala University, the subjects are "data assimilation”, “multiblock”, and “object oriented”. These words are actually the keywords for the record. So we see that different universities use subject fields in different ways. Thus, in such an open-ended research collection, it's not possible to enumerate a list of words that will include all possible computing related entries for different universities. Another factor is the language problem. Some records from a particular institution (e.g., University Library of Gerhard-Mercator University Duisburg) may list the subject(s) in languages other than English but at the same time they do have the title and abstract in English. The fourth factor is that even considering two records with the same subject, one of them might be computing related and the other may not. For example, there are a number of records harvested from Virginia Tech with the subject "Electrical and Computer Engineering". One of these records is about "high-power three-phase PWM inverters" which is definitely not computing related. Another one is about "Multicast Networks", which is computing related. In this case, we cannot get rid of all the records with such a subject. We have to look at the contents of the record to determine if it's computing related or not.

So to get better filtering results, we need to combine several sources of evidence. If an instance lacks both the contributor field and subject field, we can only rely on the content based classification as evidence of the record being computing related (i.e., text from title and/or abstract). Accordingly our filtering experiment was composed of a combination of three major steps:

- Content-based classification - classify the data set into two sets: computing related and non-computing related using title plus subject plus abstract,

- Filtering based on contributor field(s) in the metadata - enhance the results achieved through content-based classification,

- Filtering based on subject field(s) in the metadata - enhance results when records of the second step are missing contributor fields or to correct possible records mistakenly filtered out.

The experiment aimed to test: 1 ) the relation between quality of metadata and quality of services; 2) the effectiveness of content-based filtering in the absence of explicit subject and/or contributor information; and 3) the effect of combining several sources of evidence.

\section{Experimental Setting for Filtering Mechanism}

\subsection{Content-based Classification - Weka}

We have used the Weka data mining package [8] as our classification tool. Weka is a collection of machine learning algorithms for solving real-world data mining problems. It contains tools for data pre-processing, classification, regression, clustering, 
association rules, and visualization.

Classification aims to assign instances to predefined classes. Classification requires supervised learning; a training data set is used to specify the classes we are trying to learn. The performance of the classification model learned from a training set is tested on a test data set to see how well the model predicts the correct class for unseen data.

Since the collection was harvested using OAI, both the training and the test metadata were in XML files. The XML file is composed of fields like subject, title, abstract, and category for each record. The terms from title, subject, and abstract fields were used to produce the document vector for each record. Each element of the vector is a term along with its weight. Each term's weight was generated based on the tf-idf scheme. Term frequency if provides one measure of how well a term describes the document contents. Inverse document frequency (idf) measures the inverse of the frequency of a term among the documents in the collection. The motivation for usage of an idf factor is that terms which appear in many documents are not very useful for distinguishing a relevant document from a non-relevant one.

Weka expects ARFF files [9] as input. An ARFF (Attribute-Relation File Format) file is an ASCII text file that describes a list of instances sharing a set of attributes. ARFF files have two distinct sections. The first section is the Header information, which is followed by the Data information. The Header of the ARFF file contains the name of the relation, a list of the attributes (the columns in the data), and their types.

The ARFF files for our experiment were generated by parsing the XML files. All the words are listed as attributes in the ARFF file, and the document vector along with the category of each document constitute the Data part of the ARFF file.

Part of an example header on our training data set is:

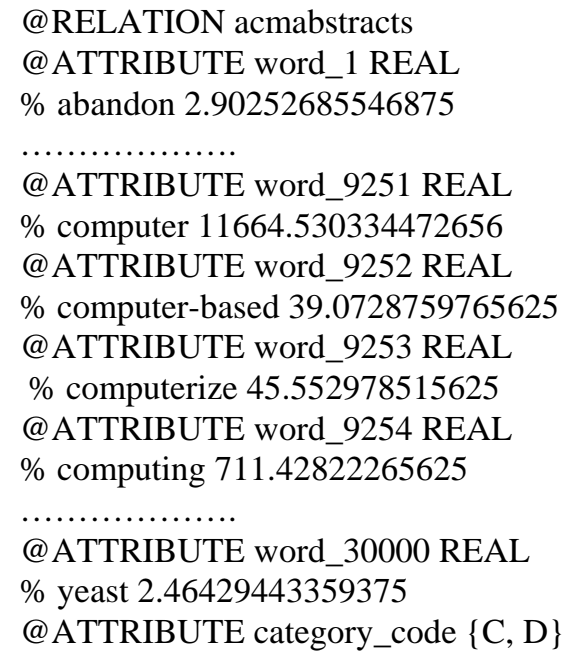

The list of the attributes is composed of the words (stop words removed) and an enumeration of the category for each record. Each word attribute's value is a real number which is that word's total frequency in the collection. Each line that begins with a "\%" is a comment line explaining what the word is and its total frequency in 
the collection. The category "C" represents a computing related record, and the category "D” represents a non-computing related record.

Part of the Data of the ARFF file is the following:

\section{@DATA}

\{2545 0.04974365234375, 2867 0.14984130859375, 5890 0.19989013671875, 30000 “C” $\}$

\{222 0.0372314453125, $19510.0372314453125,25450.0372314453125,30000$ “D”\}

The Data part contains several rows. Each row represents a document and is surrounded by curly braces. The format for each entry is: <index $><$ space $><$ value $>$. Here index is the attribute index (starting from 0). For example, in the first row of the above example, “2545” is the attribute index 2545 (word 2546) and its value is a real number which is that word's weight computed through using $t$-idf. The last entry of each row is the attribute "category_code”, which is the category of this document.

We chose to use the WEKA SMO [10] classifier. The SMO classifier implements the sequential minimal optimization algorithm. It is a fast method to train Support Vector Machines (SVMs) which has reportedly been the best classifier so far for text classification experiments [11-15]. The main idea of Support Vector Machines is that given a set of data points which belong to either of two classes, an SVM finds the hyperplane that leaves the largest possible fraction of points of the same class on the same side and maximizes the distance of either class from the hyperplane. The optimal separating hyperplane will minimize the risk of mis-classifying the training samples and unseen test samples. Figure 3 illustrates this idea.

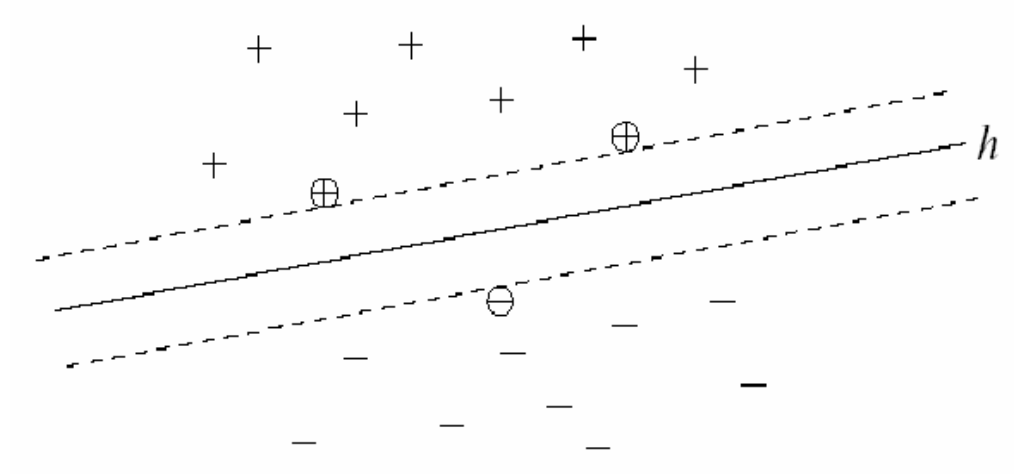

Fig. 3. Support Vector Machines (SVMs) find the hyperplane $h$, which separates the positive and negative training examples with maximum distance. The examples closest to the hyperplane are called Support Vectors (marked with circles)

Figure 4 shows the composition of the training and test sets for the experiments. Normal arrows represent how we selected the training collection and the test collection. Dotted arrows represent the input to the training and classification process. 
Dashed lines represent the output of the training process.

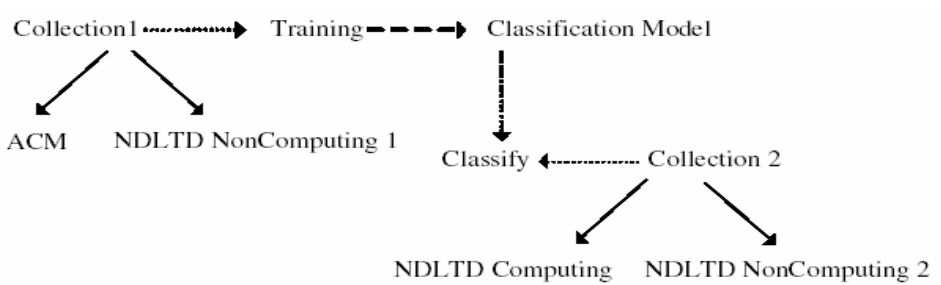

Fig. 4. Training set and Test set composition

For the experiments, the metadata harvested from the Union Catalog were manually divided into two categories for test purposes: computing related and others. We used records extracted from the ACM Digital Library (included in CITIDEL) to provide positive training examples to our classifier instead of some other computing related records because the previously identified computing sub-collection in NDLTD only has 138 entries. We felt this sub-collection was too small to serve as the computing category of the training set. Since we need to separate the computing related entries from the remaining records, all the ACM metadata was marked as one category computing related. Half of the non-computing related entries from the Union Catalog served as the non-computing category of the training set. All the computing related entries and another half of the non-computing entries served as the test collection.

\subsection{Contributor Filtering}

To remove false computing entries from the classification result set, we decided to look further at the contributor fields for those classified as computing entries. For an ETD, the contributors are normally members of the committee. A simple heuristic was defined wherein if more than half of the contributors are computer scientists, this thesis or dissertation is assumed to be computing related.

We used a set of author names extracted from records of the ACM DL as our computer scientists list. When we match a name, different formats need to be considered. For example, we may have 'Edward A. Fox', but the contributor name might be in the format of 'Edward Fox', or 'E. A. Fox'. Even if the name in the computer scientists list doesn't exactly correspond to the contributor name, we should count this case as a match. If an exact name match fails, the mechanism we used was:

- If their middle initials are the same, we compare the initials of their first names and the whole last names. For example, if the contributor is in the format "E. A. Fox" and the computer scientist is in the format "Edward A. Fox", both the initials of the first name is "E" and both have the same last name, so this is counted as a match. 
- If and only if one of the two compared names' middle is empty, we compare their first names and last names. For example, if the contributor is in the format "Edward Fox" and the computer scientist is in the format "Edward A. Fox", they have the same first name and last name, so this is counted as a match according to this rule.

In our experiment, we tried to match 495 contributor names with the names in the computer scientists list (extracted from the ACM DL). 277 of the 495 were matches. Among these 277, 156 had exact name matches. In addition, 35 are matched based on the first scheme above, and 86 are matched based on the second scheme above.

\subsection{Subject Filtering}

From the analysis of NDLTD records, it was noted that contributors were not used uniformly and consistently across institutions (recall Sect. 1.1). Therefore many entries may not be filtered using only the contributor filter. For example, we noticed that 88 of 129 non-computing related entries were missing contributor fields in their metadata. So to deal with this situation, we applied a second filter on those entries missing contributor fields. We analyzed the metadata of those entries and found that 71 of 88 entries have subject fields. We noted that some non-computing words are subjects. For example, words like 'physics', 'animal science', 'Landscape Architecture', etc. appear. So we could use them to remove entries whose subject field had such kinds of words.

We also noticed that when the contributor filter was applied on the classified computing collection, a few positive entries were filtered out. So we also applied the subject filter in another way on these entries. That is, we check if these entries' subject field contains words like 'Computer Science' and if they do, we move them back into the computing collection.

We choose to pick contributors before subject(s) because we feel that contributor information is more reliable. Due to the inconsistency in use of subjects by several universities as indicators of field, it's hard to choose several words and say that records with such words in the subject field are computing related but otherwise are not computing related. So it's a better choice to use subject filtering as a supplement to the content based filtering and contributor filtering, to improve filtering accuracy.

\section{Experimental Results}

\subsection{The content-based classification result was as follows:}

We have two classes in our classification. One is " $\mathrm{C}$ " which represents the computing related (positive) instances and another is " $D$ " which represents the noncomputing related (negative) instances. The total number of instances classified by the classification system in the test set was 3792, whereas 138 were positive instances and 3654 were negative instances. 96.3\% (3652) of the total instances were correctly 
classified while 3.7\% (140) were incorrectly classified.

Table 1 gives the detailed accuracy for these two classes. Table 2 shows information about actual and predicted classifications of the system.

Table 1. Detailed accuracy by class

\begin{tabular}{llll}
\hline Class & Precision & Recall & F-Measure \\
\hline C & 0.496 & 0.920 & 0.645 \\
D & 0.997 & 0.965 & 0.981 \\
\hline
\end{tabular}

Table 2. Content-based classification result

\begin{tabular}{lll}
\hline Actual Class & Classified as C & Classified as D \\
\hline C & 127 & 11 \\
D & 129 & 3525 \\
\hline
\end{tabular}

\subsection{Contributor filter results}

To remove those 129 entries which are classified as computing related entries but actually are non-computing entries, we run the contributor filter on the classified computing collection through the content-based classification system. Among those 129 entries, we were able to filter out 39 entries. This is mainly because 88 entries of those 129 don't have contributor fields. 2 non-computing entries failed to be filtered out because more than half of their contributors were in the computer scientists list that we extracted from the ACM DL. One of these two is a dissertation with the title "Numerical Differentiation Using Statistical Design" in the field of Statistics from North Carolina State University (NCSU), and the other is a dissertation with the title "Autonomous Solution Methods for Large-Scale MARKOV Chains" in the field of Operations Research from NCSU. We also filtered out 17 actual computing entries. We want to move back those 17 entries. To deal with the errors in filtering and missing contributor entries, we applied the subject filter on those entries.

\subsection{Subject filter results}

Among those 88 missing contributor entries, we were able to filter out 65 through the subject filter. 17 entries don't have subject fields. 6 of them failed to be filtered out by the subject filter because their subject contains words where it is hard to say if they are computing related, for example, phrases like "library and information sciences", "Electrical and Computer Engineering”, etc.

We applied a subject filter on the 17 computing entries filtered out by the contributor filter. We successfully moved 16 of them back to the computing collection. 


\section{Evaluation}

Table 3 shows the experimental results in terms of the changes of the number of positive and negative instances after each step of the experiment, and the number of positive and negative instances in the final computing collection.

Table 3. Experimental Results

\begin{tabular}{lll}
\hline & Computing & Non-Computing \\
\hline Content-based classification & 127 & 129 \\
Contributor filter & -17 & -39 \\
Subject filter & +16 & -65 \\
Final computing collection & 126 & 25 \\
\hline
\end{tabular}

For completeness, we also tested the result of applying only a subject filter to the results achieved through the content-based classification. All the experimental results are summarized in the recall and precision measures in Figure 5. Precision is defined as the proportion of correctly classified records in the set of all records assigned to the target class. Recall is defined as the proportion of correctly classified records out of all the records having the target class.

Content based classification yields good recall but low precision. After applying a subject filter to the content based classification, we improved the precision without loosing recall. When we applied a contributor filter to the content based classification result, we also improved precision but at the same time filtered out some computing entries, so recall was reduced. On the other hand, after applying the subject filter, we could remove more false computing entries and the same time bring back some real computing ones. So considering both recall and precision, we achieved the best results by combining these three types of evidence.

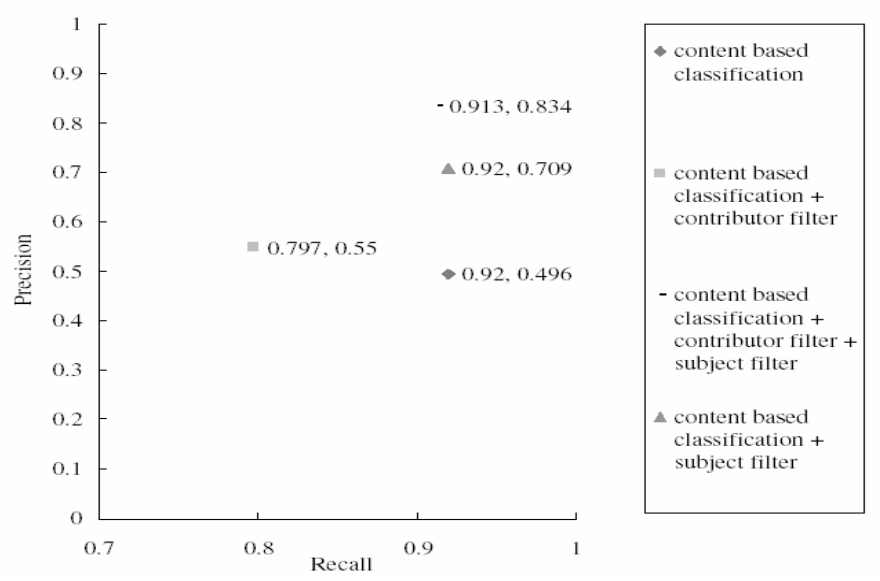

Fig. 5. Experimental results in Precision Recall format

We also calculated the F-Measure for each step of the experiment. F-measure is 
defined as $2 *$ Precision*Recall / (Precision + Recall). Table 4 shows the values of the F-measure for each step.

Table 4. F-Measure values for each experiment step

\begin{tabular}{ll}
\hline Experiment & F-Measure \\
\hline Content-based classification & 0.645 \\
Content-based classification + contributor filter & 0.651 \\
Content-based classification + subject filter & 0.801 \\
Content-based classification + contributor filter + subject filter & 0.872 \\
\hline
\end{tabular}

\section{Conclusion}

We have presented a set of experiments to extract computing related theses and dissertations from the NDLTD Union collection to include in the CITIDEL collection. Our experiments show that the quality of the filtering service was affected by the quality of the metadata. If the metadata contains more fields and if the fields were consistently used across all NDLTD data providers, we can utilize those fields to improve filtering. Several sources of evidence were combined to overcome the problem of inconsistency of the instances' metadata and to yield better results. To address the problems of absence, inconsistency, and ambiguity of contributor and subject information in NDLTD, content-based classification was studied. This procedure gives good recall but low precision. The contributor filter tried to look at the contributor field(s) and to filter out non-computing related instances. And to address the problem of lacking contributor field(s) for some instances, the subject filter tried to look at the subject information to filter out non-computing related instances. The final combination of the filtering mechanisms provided satisfactory precision and recall.

One area of future work is to enhance the content-based classification result by trying to apply different weights to the words in the title, subject, and abstract fields to reflect their relative importance. Another area of future work is to combine other sources of evidence like the department field in other formats of metadata like ETDMS (the recommended metadata standard for ETDs), when it is available, or the full-text of the ETD for the content-based approach. We also will formalize and extend our filtering methods by using probabilistic tools like belief networks to combine multiple sources of evidence. Finally we plan to apply similar strategies and methods to filter electronic theses and dissertations in many other fields covered by NDLTD (e.g., Physics, Mathematics, etc.).

\section{Acknowledgements}

This research work was funded in part by the NSF through grants DUE-0136690, DUE-0121679 and IIS-0086227. The second author is also supported by CAPES, process 1702-98 and a fellowship by American Online (AOL). 


\section{References}

1. Fox, E.A.: Computing and Information Technology Interactive Digital Educational Library (CITIDEL). Homepage (2002). http://www.citidel.org/

2. Fox, E.A.: Networked Digital Library of Theses and Dissertations. Nature Web Matters (1999)

http://helix.nature.com/webmatters/library/library.html

3. Fox, E.A.: Networked Digital Library of Theses and Dissertations (NDLTD). Homepage (1999) http://www.ndltd.org

4. Suleman, H., Atkins, A., Gonçalves, M.A., France, R.K., Fox, E.A., Virginia Tech; Chachra, V., Crowder, M., VTLS, Inc.; and Young, J., OCLC: Networked Digital Library of Theses and Dissertations: Bridging the Gaps for Global Access - Part 1: Mission and Progress. D-Lib Magazine, 7(9) (2001)

5. Suleman, H., Luo, M.: Electronic Thesis/Dissertation OAI Union Catalog. Homepage (2002) http://rocky.dlib.vt.edu/ etdunion/cgi-bin/index.pl

6. Van de Sompel, H.: Open Archives Initiative. WWW site. Ithaca, NY: Cornell University (2000) http://www.openarchives.org

7. DCMI: Dublin Core Metadata Element Set, Version 1.1: Reference Description. Available from http://www.dublincore.org/documents/dces/

8. Witten, I.H., Frank, E.: Data Mining: Practical Machine Learning Tools and Techniques with Java Implementations. Morgan Kaufmann, San Francisco, CA (2000)

9. Paynter, G.: Attribute-Relation File Format (ARFF). WWW site. http://www.cs.waikato.ac.nz/ ml/weka/arff.html

10. Platt, J.: Fast Training of Support Vector Machines using Sequential Minimal Optimization. Advances in Kernel Methods - Support Vector Learning, B. Schölkopf, C. Burges, and A. Smola, eds., MIT Press (1998)

11. Joachims, T.: Text categorization with support vector machines: learning with many relevant features. In Proceedings of ECML-98, 10th European Conference on Machine Learning (Chemnitz, Germany, 1998), 137 - 142

12. Dumais, S. T., Platt, J., Heckerman, D., and Sahami, M: Inductive learning algorithms and representations for text categorization. In Proceedings of CIKM-98, 7th ACM International Conference on Information and Knowledge Management (Bethesda, MD, 1998), 148 - 155

13. Joachims, T.: A statistical learning model of text classification for support vector machines. In Proceedings of the 24th annual international ACM SIGIR conference on research and development in information retrieval (New Orleans, Louisiana, 2001), 128 - 136

14. Dumais, S., Chen, H.: Hierarchical classification of Web content. In Proceedings of the 23rd annual international ACM SIGIR conference on research and development in information retrieval (Athens, Greece, 2000), 256 - 263

15. Sebastiani, F.: Machine learning in automated text categorization. ACM Computing Surveys (CSUR), Vol. 34, Issue 1 (2002), 1 - 47 